\title{
Modeling as a Method of Describing the Lexical Meaning Structure
}

\author{
O. Kistanova \\ Foreign Languages Department \\ Samara State Technical University \\ Samara, Russia \\ sq.olga@mail.ru \\ V. Savitskiy \\ Foreign Languages Department \\ Samara State Technical University \\ Samara, Russia \\ Lampasha90@mail.ru
}

\author{
V. Dobrova \\ Foreign Languages Department \\ Samara State Technical University \\ Samara, Russia \\ victoria_dob@mail.ru \\ E. Gashimov \\ English Philology Department \\ Samara branch of Moscow State Pedagogic \\ University,Samara, Russia \\ elchgash@mail.ru
}

\author{
L. Nurtdinova \\ Samara State Technical University \\ Samara, Russia \\ liliandrr@gmail.com
}

\begin{abstract}
The paper deals with the issue of modeling the structure of lexical meaning from the perspective of modern semantics. The analysis of the existing theories is carried out in order to stress the importance of using this method. The correlation of language units with the reality which is fixed in their lexical meaning is described. Modeling is the method that helps us explain that correlation and describe components of lexical meaning in detail. Characteristic features of linguistic models are shown, and on this basis their classification is presented. The main principle of the classification is the opposition of models due to different aspects. The specific of the term 'modeling' is cleared up, and concepts of an object level and a meta-level are differentiated. The difference between 'a language model' and 'a linguistic model' is explained. As a result, an example of modeling the lexical meaning of a word is given in order to emphasize the practical application of the modeling method.
\end{abstract}

Keywords-modeling; lexical meaning; language unit; metalanguage; characteristic features

\section{INTRODUCTION}

The correlation between the meaning of a language unit and the part of reality it denotes has been studied by linguists for a long time, yet one cannot consider it to have been completely explored by now. Undoubtedly, most of the meaningful language units correlate with the reality, but the question about the information which creates the meaning of a sign is still unclear, and the terms used to denote components of this information are also very diverse. In this regard, it is interesting to consider modeling [2-20] from the point of view of modern semantics, to organize basic terminology and structural characteristics of the models and to determine the place of the modeling method to describe the structure of lexical meaning.

The research of 'the plane of content' of a language is an extremely difficult task because of an originality of that object which lies beyond the scope of direct observation and affects underexplored spheres of mental activity of a person. The results of application of various ways of lexical unit semantics description demonstrate that semantics can't be described in the available ways in all its completeness and variety, as in this case, the artificial metalanguage would have to surpass the object language (the natural language) in a number of characteristics.

Therefore, researchers set a more precise task: to describe the semantics of lexical units in an abstract way, to reflect only the main system relations between the semes (which make a lexical meaning), to reproduce its relational framework into which the content is placed. Such description represents a more or less precise part of a lexical meaning and its characteristics correspond to the definition of a model.

In all scientific areas where an object of research is inaccessible to direct observation, there is a need for modeling. In such cases, as a rule, the object of research assimilates to a certain 'black box'; one knows what initial materials are placed in it and what final products come out of it at the end.

The technique of 'a black box' has been proposed as one of research methods in cybernetics, but due to its flexibility, it has been used in all researches where a direct observation of 
The term model is used in linguistics quite often, however, it has different meanings. In linguistics the following interpretations are most widely used:

an abstract concept of a standard or a sample of any system (grammatical, lexical, phonologic, etc.);

a representation of the most general characteristics of a language phenomenon;

a general scheme of description of a language system or its subsystem [4];

a scheme or a sample of a unit showing a consecutive arrangement of its parts [9].

While describing a naive view of the world, linguists use the term model in the meaning - a fragment of the linguistic view of the world reflected by language means, and a way of representation of any category in the language. In some papers, a model is considered to be an interpretation of a fragment of reality which is embodied in word semantics; it is thought to be not a result but a means of cognition, i.e. a cognitive tool - the system of signs playing a role in the information representation (or coding) and its transformation. J. Neuymin offers the following generalized structure of a scientific model: The model is a four-part structure whose components are the subject, the task (solved by the subject), the object-original and the language of description (or the way of material reproduction) of a model. A special role in structure of a generalized model is played by the task which is solved by the subject. This task plays the role of the main factor in the process of integration of object's properties into a uniform integrated image (as some kind of system) [13]. From this fundamental definition of a structural model, one can deduce some of its properties:

- a model/modeling always assumes the presence of some active subject (creator) who reproduces characteristics of reallife objects in the objects he creates;

- each object-original corresponds to a number of models which perform some definite tasks (besides, performing a task also assumes some variability), therefore a model corresponds to the object-original only partly;

- as a result of his interpretations, a researcher comes to the conclusion that the fact that scientific concepts which have been developing historically and which are connected with objective reality directly or indirectly (laws, theories, scientific views of the world) are, in fact, modelled constructions. All our scientific knowledge in the field of environment, society and technology, all information about ourselves, about human thinking processes and their regularities are made in a modelled way [11].

The characteristic features of models, thus, are the following:

1. Subjectivity: a researcher makes a selection of properties in which the model corresponds to the original.

2. Compactness: models reproduce an object in a simplified form, therefore a model is always more compact than the original. A more versatile and effective description of 
reality requires a set of models, or complex, multi-level models.

3. Transformability: with the model it is possible to make what can't be made with the original. Such possibility of transformations is the most fundamental and informative idea of a method of modeling, therefore the model is used to research objects that are difficult or impossible to research due to the ethical or organizational reasons.

4. Dual nature: in the perception process, a model itself replaces an object, thus keeping some characteristics that are important for a researcher, and itself becomes an object of a direct research. In such a way, a model is at the same time both a research object, and means of perception.

5. Specific informational content of a model as a means of perception: a model always exists in an abstract form, therefore the properties which are not presented in the original are distinguished in it [10].

From the above mentioned definitions one can deduce the following essential categorial characteristics of a model:

1) a model is a research object substitute - quasi-object created with the purpose of studying an object;

2) a model is capable of providing the information on an object;

3) a model, in contrast to a copy of an object, reproduces an object incompletely - only one of its aspects;

4) a model is an analogue of the corresponding object;

5) a model has such characteristics as compactness and vivid visual representation, and this fact gives us an opportunity to study an object in all its aspects [15].

As V. Shtoff mentions, the difference between a model and a theory lies in a way of reflection of an object. While a theory content is a set of separate judgments connected by logic laws and by special scientific laws, a model content is some typical situations, structures, sets of idealised (i.e. simplified) objects, etc. in which the laws formulated in a theory are carried out. Therefore, a model is always some certain construction, evident in this or that way, and capable of being observed [16].

\section{Methodology}

Having considered models from different angles, the authors designed their classification.

According to the way of creation, all models are divided into material and ideal ones. Material (substantive) modeling is based on material analogy of an object and its model. To create models of this type it is necessary to select functional characteristics (geometrical, physical) of the object studied. An example of such substantive models is socialpsychological experiments.

A large class of models is represented by the ideal models based on mental analogy. Ideal models are mental constructions, some theoretical schemes, images of original objects which are not necessarily intended for the subsequent physical embodiment. The objectivization of such mental constructions in the form of graphs, texts, figures, etc. doesn't turn them into material ones, as these models exist and function only in a person's imagination, and written texts, graphs, etc. are only an explicit sign expression of cognitive processes.

Ideal modeling, in turn, is subdivided into sign (formalized) modeling and intuitive one. Intuitive modeling is used at the beginning of a cognitive process when connections within a system are very complex. Life experience of a person (which can be considered an intuitive model of interpersonal relations) can be an example. Models of sign modeling are language models, graphs, drawings, schemes, formulas. The most important type of sign modeling is mathematical and computer modeling.

A mathematical model is an abstract system consisting of a set of mathematical objects [8]. They, in the turn, can be of two types: description models (imitation models) and explanation models. The compliance between a formal and physical structure which is not caused by some rule is a characteristic feature of a description model. A description model presents another type of informative models where the structure of an object is in compliance with its mathematical representation and it can be easily explaned.

Computer models are based on programming with the use not of the equations (as mathematical models do) but with the use of algorithms, i.e. strictly formulated consecutive instructions. These models are especially effective while studying the situations connected with processing of a large amount of information. Such form of a computer model as an expert system using the a number of 'if ... then...' programmes is very often applied" [10].

As a rule, ideal models are subdivided into figurative ones which are constructed on the basis of sensual-visual elements and which are implemented the form of schemes, drawings, figures, and sign ones in which the connection between signs and their meanings is of spontaneous character. There also exist mixed models (which can also be called figurative-sign models) [15].

According to the reproduced characteristics of the original, all models are divided into structural (which reproduce the structure of the original in a general way), substantional (which have substance, system elements and material similar to the original) and functional models (which act in the environment in a similar way with the original).

One of the most important concepts of the theory of models is analogy which is defined as 'similarity in the relation between two objects, i.e. ... similarity which is based not on separate properties or parts of the objects, but on interrelations between their properties or parts' [16]. Analogy is useful and important for a method of modeling, since analogy-based relations form the method of modeling restrictions. As a model - as means of cognition - is based on analogy (metaphorical, cognitive, graphic, system one), it loses its sense in a case of identity of a model and a prototype, on the one hand, and also in a case of a big difference between an object-original and its model, on the other hand. 
Analogy acts in a form of isomorphism or homomorphism. The isomorphism is mutual compliance between structural elements of an original and a model, the homomorphism is a less exact, approximate compliance between them. Homomorphism is a more general and a weaker relation between a model and its prototype when one of the three conditions is not met: function compliance; elements compliance; one-to-one compliance of properties and relations.

On the contrary, the relations between a model and its prototype are isomorphic if all these three conditions are met. The isomorphism can be considered as a limit, an extreme case of homomorphism [16]. Taking into account the abovementioned facts, one can say that a model is an isomorphic or homomorphic image of the object-original which is being examined [8].

The concept of analogy is especially important in those cases when the original and its model have different ontologic nature - for example, a fragment of non-linguistic reality and its linguistic model.

An ideal model is a synthetic form which acts as an intermediate link between sensuality and cognition, between a visual perception and representation of phenomena and nonvisual representation of their entities [16] in judgments and theories.

As E. Stewart mentions, a model is a step on the way to a theory which is capable to form a character of the theory. A model can specify possible ways of development of the theory and the additional fields of its application [17]

Taking into account the above mentioned classification, it can be said that the term 'modeling' is used in three basic meanings when one speaks about language units:

1) a structure and creation of language units on certain samples and standards; units;

2) a specific representation of reality by means of language

3) a scientific description of a structure, creation and functioning of language units in compliance with the methodological principles stated above.

The first meaning reflects the correlation between a language sign and his denotation, the second shows a standard correlation between a sign and its meaning, the third demonstrates a correlation between a sign (as an object of a research) and a metalanguage of its description. Although these three meanings are interconnected, in V. Savitsky's opinion, they should be differentiated accurately to avoid a confusion of the concepts relating to an object level and a meta-level [15].

In the English language there are terms a language pattern and a linguistic model which allow to carry out such a differentiation. Similarly, let us distinguish the terms $a$ language model (a natural language sample on the basis of which a number of language constructions are constructed) and a linguistic model (a theoretical construction describing any language phenomenon (including a language model)).
There is no contradiction. In fact, analogy is symmetric: when two phenomena are homomorphic, each of them is a model of the other. It is a function of a model that has a great importance. Having been built as a reconstruction of an object-original, a model then can itself become an original or a pattern for creating similar (analogous) objects. Linguistic models which are created as copies of language models can later become the patterns which can help us predict and imitate the functioning of language models. To sum up, linguistic models reflecting language models functioning and structure are, in fact, metamodels, or models of models (models of patterns).

Let us examine in brief the general principles of creating linguistic models. U. Apresyan proposes the following important properties of linguistic models:

1) it is possible to model only such phenomena which essential properties are limited by their structural (functional) characteristics and which are not connected with their physical characteristics in any way;

2) a model doesn't deal with the concepts of real objects but with their constructs, i.e. with the concepts about ideal objects which are not based directly on experimental data, but are constructed on the basis of some general hypotheses which are the result of observations and a researcher's intuition;

3) a model is always some kind of idealisation of an object;

4) any model has to be formal, i.e the initial objects connecting statements and rules of operating them have to be set in an obvious way;

5) any model has to have explanatory possibilities [1].

Finally, a linguistic model is considered to be an ideal structural and/or functional model. It can combine characteristic features of a figurative model (if it contains visual presentation, i.e. graphs, drawings, schemes, etc.) and a non-figurative model (if it does not include any visual presentation).

\section{EXPERIMENT}

A language of a word's lexical meaning description should meet the following requirements. Firstly, it should be universal, i.e. it should be able to express accurately a variety of meanings. Secondly, it should have procedural components; and then it will be possible to build the models of cognitive processes of natural language perception on this basis and implement them in computer programs. Thirdly, such language should be quite natural and easy-to-understand [18].

In order to achieve these goals, the artificial formalized meta-languages, which are also referred to as formalized systems, logic systems or systems of formal logic etc., are used. The creation of such artificial language of logic begins with building its alphabet which consists of elementary symbols: logical symbols (signs of logical operations and relations, for example, propositional connectors and quantifiers), non-logical symbols (parameters of descriptive components of a natural language) and technical symbols 
(such as brackets). Then the so-called rules of formation of complex language signs out of simple ones are formulated, i.e. various types of well-formed expressions (the most important type of which are formulas, i.e. the analogues of natural language sentences) are set.

The advantage of a formalized description over the natural language description is that it is a) unified, b) reduced, c) generalized. It is identical for all situations, and it reveals their invariant structure which can not be seen when comparing the numerous descriptions in the natural language. In cognitive linguistics, a number of sign systems which are more or less formal (i.e. reduced/unified/generalized) and based on various methodologies (symbolic logic, graph theory, situational semantics) were developed. These include the so-called declarative and procedural semantic representations, semantic networks as well as cognitive structures that are also called frames, scripts, scenarios.

Having taken into account everything mentioned above, the authors developed an artificial semantic metalanguage called AFFECT, which is used to describe the concepts of human emotions [4].

Like any formalized sign systems, it includes an information component (a set of units (glosses) which is called a glossary) and a procedural component (different rules of units combination while composing texts (scripts)). The glossary of the AFFECT metalanguage contains the following types of units: terms, event variables, operators, quantifiers and logical connectives.

Terms are glosses which name corresponding nouns. For example: $\mathrm{i}$ - an instrument of action (Latin instrumentum), $\mathrm{t}$ time (Latin tempus), etc. The letters $x, y, z$ represent animate 'actors' of the situations described; other letters (for example, o - "object", v - "value", etc.) stand for inanimate actors.

Event variables indicate the influence of actors on some other actors.

Operators are divided into predicate, modal and evaluative ones. (Predicate operators: THERE IS, HAS, PERFORMS; modal operators: IT IS POSSIBLE THAT, IT IS PROBABLE THAT; evaluative operators: IT IS MORAL THAT, IT IS GOOD THAT, etc.).

There are two quantifiers: the existential quantifier (for SOME objects of class $x$ IT IS TRUE THAT ...) and the universal quantifier (for ALL objects of class $x$ IT IS TRUE THAT ...).

There are also some logical connectives, e.g. conjunction (and) and disjunction (or).

Let us give an example of modeling a lexical meaning using the AFFECT metalanguage for the word 'joy'. Its model looks like this:

JOY: FEELS $+x$ (WANTS $x$, A AND ANTICIPATES $x$, NOT A AND PERCEIVES $x, A)$,

where $x$ is an actor of the situation, A is a desired state of things, and FEELS, WANTS, etc. are operators denoting emotions (positive or negative) and actions.
The authors work out a scenario in which this model is further developed:

\section{THE SCENARIO OF JOY / TRIUMPH}

1) INITIAL SITUATION: THERE IS $x$. THERE IS z.

2) COGNITIVE STATE OF $x$ : DOESN'T PREDICT $x$ (PLEASES $z, x$ ); alternative: BELIEVES $x$ (IT IS UNLIKELY (PLEASES $z, x)$ ).

3) ACTION OF z: PLEASES z, $x$.

4) PERCEPTUAL STATE OF $x$ : PERCEIVES $x$ (ACTION $O F z)$.

5) RESULT: (EMOTIONAL STATE OF $x$ ): REJOICES / EXULTS $x$ (IS PLEASED $x)$.

The actor $\mathrm{z}$ (the one which gives pleasure) is not necessarily a subject; it can be a natural phenomenon, a combination of circumstances, destiny - i.e., good luck.

A characteristic feature of this emotion is that it can be considered (unlike content, satisfaction, etc.) to be a reaction to an unexpected pleasant event or, at least, to such an event which the subject thought to be hardly possible. Therefore, sharpness of this emotion is of sharp and 'explosive' character. This fact is reflected in the scenario (in line 2).

The reason for feeling joy / triumph is good luck. The expression 'good luck' is defined in Concise Oxford Dictionary as follows: 'chance as bestower of good fortune, fortuitous event bringing success'. In spite of the fact that in this definition the seme [chance] appears twice (in word meanings - chance and fortuitous), it is necessary to remember that good luck and joy usually characterize those people who persistently try to obtain them.

Now let us turn to analysing a meaning of the lexical unit which is a designator of a concept of the above-mentioned emotion.

The word joy is defined in Concise Oxford Dictionary as 'vivid positive emotion aroused by pleasant surprise'. It is the only analytical definition of this word the authors found to form the basis for designing the scenario of the corresponding concept. In our opinion, other definitions of this concept have mainly the synonymic character complicating identification of semantic originality of this lexical unit. If the word $A$ is defined by the word $\mathrm{B}$, and the word $\mathrm{B}$ is defined by the word $\mathrm{C}$, and the word $\mathrm{C}$, in turn, is defined by the word A, there is a circulus vitiosus (a vicious circle in definition) that makes it difficult to identify the specifics of this lexical unit.

Nevertheless, the authors have tried to take as much information as possible from dictionary definitions. So, in the above-mentioned definition of the word joy categorial characteristics of the corresponding concept are the following: it is an emotion (emotion); it is positive (positive); it has the reason (aroused by) which consists in pleasant astonishment (pleasant surprise). In turn, the word surprise is defined as 'emotion excited by the unexpected'. It, in our opinion, is the most detailed characteristic of joy. 
In this model, the whole situation in which this emotion appears is reflected, and the characteristic features of the word's lexical meaning are shown.

\section{DISCUSSION}

To sum it up, modeling is an effective method of perception and comprehension of the real world developed by a person in the course of the evolution. This method is applied to both scientific field and everyday practice. The results of its usage are recorded in theoretical and applied scientific models, literature texts and in a language system. Using the modeling method for description of the structure of a word's lexical meaning can be a very promising way of development in the sphere of modern linguistics

\section{References}

[1] U. D. Apresyan,. Ideas and methods of modern structural linguistics. Moscow: Prosveschenie, 1966, 302 p.

[2] A. Brock, Modelling the complexity of humour - Insights from linguistics. Lingua. In Press, Corrected Proof. Available online 13 May 2017.

[3] Jasper C. van den Herik, "Linguistic know-how and the orders of language," Language Sciences, vol. 61, May 2017, pp. 17-27.

[4] V. V. Dobrova, O. A. Kistanova, "Meta - languages: nature and characteristic features," Issues of cognitive linguistics, No.1, pp. 114117, 2017.

[5] S. Fleischman, "Discourse as space/discourse as time: Reflections on the metalanguage of spoken and written discourse," Journal of Pragmatics. vol. 16, Iss. 4, pp. 291-306, October 1991

[6] F. Gobbo, "Are planned languages less complex than natural languages?," Language Sciences, vol. 60, pp. 36-52, March 2017.

[7] M. H. van Velzen, L. Nanetti, P. P.de Deyn,"Data modelling in corpus linguistics: How low may we go?," Cortex, vol. 55, pp. 192-201, June 2014.
[8] S. A. Lebedev, The science philosophy. Moscow: Academic project, 2007, 731 p.

[9] A. F. Losev, Introduction in general language models theory. Second edition. Moscow: Editorial URSS, 2004, 296 p.

[10] L. V. Minaeva, Speech communication in politics. Moscow: Flinta, 2007, 248 p.

[11] N. A. Mishankina, The issues of modeling in a language and speech. A metaphor in science: a paradox or a standard? Tomsk: Tomsk university publishing, 2010, pp. 8-56.

[12] R. M. Nefdt, "Linguistic modelling and the scientific enterprise," Language Sciences, vol. 54, pp. 43-57, March 2016.

[13] J. G. Neuymin, Models in science and engineering: history, theory and practice. Leningrad: Nauka, 1984, 189 p.

[14] I. I. Revzin, Language models. Moscow: Nauka, 1962, 192 p.

[15] V. M. Savitsky, English phraseology: issues of modeling., Samara: Samara University, 1993, $172 \mathrm{p}$.

[16] V. A. Shtoff, Modeling and philosophy. Moscow-Leningrad: Nauka, 1966, $301 \mathrm{p}$.

[17] A. H. Stewart, Graphic Representation of Models in Linguistic Theory. Bloomington and London: Indiana Univ. Press, 1976, 195 p

[18] V. D. Solovjov, To creation of meta-language of semantics description . Kazan: Otechestvo, 1999. pp. 75-84.

[19] J. Talbot, "Taylor Languaging, Metalanguaging, Linguistics, and Love," Language Sciences, vol. 61, Pages 1-4, May 2017.

[20] Z. Zhang, Ya. Sun, G. W. Humphreys, Ya. Song, "Different activity patterns for action and language within their shared neural areas: An fMRI study on action observation and language phonology," Neuropsychologia, vol.99, pp. 112-120, May 2017. 